\title{
Potential Roles of Extracellular Traps in the Progression of Coronavirus Disease (Covid)-19
}

\author{
Kartika R Pertiwi ${ }^{1 *}$ \\ ${ }^{I}$ Department of Biology Education, Faculty of Mathematics and Natural Science, Universitas Negeri Yogyakarta, \\ Indonesia \\ "Corresponding author. Email: kartika.pertiwi@uny.ac.id
}

\begin{abstract}
Millions of people have suffered from coronavirus disease (COVID)-19 worldwide with diverse clinical presentations, ranging from asymptomatic to severe pneumonia leading to respiratory failure and even death. In critical phases, thrombosis and inflammation are considered as the important contributors in the processes. Extracellular traps (ETs), meshes-like chromatin DNA decorated with antimicrobial proteins, have become interesting features in immunothrombosis research due to their pro-inflammatory, pro-thrombotic and pro-coagulant properties. These traps, released by innate inflammatory cells, mark a distinct form of cell death namely etosis. Whether etosis may also participate in the thrombotic inflammation of Covid-19 progression and whether the roles are beneficial or detrimental are still not much known. This review presents current knowledge on the formation, properties and roles of ETs in human non-infectious diseases, particularly in inflammation, thrombosis and coagulopathy, followed by an exploration on how SARS-CoV2 may trigger the formation of ETs. Next, the pathogenesis of Covid-19 is briefly discussed, gathering current findings on the involvement of ETs in the immunopathological mechanisms during Covid-19 infection. Lastly, this review discusses the potential roles of etosis in the progression of Covid-19, thereby targeting the extracellular traps as a severity biomarker and/or a therapeutic approach in the management of Covid-19.
\end{abstract}

Keywords: Extracellular Traps, Etosis, Thrombosis, Inflammation, Coronavirus.

\section{INTRODUCTION}

The pandemic of Coronavirus disease (Covid)-19 caused by severe acute respiratory syndrome coronavirus 2 (SARS-CoV-2) is still currently ongoing in several countries, including in Indonesia. Although some countries have declared their new normal phase, the second wave of Covid-19 indeed occurs and possesses a threat. The improvement of clinical management and treatment for Covid-19 has succeeded to cure more patients with less sequelae effects. Nevertheless, in some cases, Covid-19 complications have been reported to be more diverse, dynamics and also dangerous. Not only severe pneumonia leading to acute respiratory distress syndrome (ARDS), but also cytokine storm [1], thrombosis and thromboembolism [2,3], coagulopathy [4], sepsis and multiple organ failures [5], are reported to be the cause of death in Covid-19 patients.

Recent clinical and autopsy reports on the association of thrombosis in Covid-19 cases with complication have drawn attention from clinicians and researchers worldwide [2,6]. It is estimated that thrombotic complication may affect up to 50\% confirmed Covid-19 patients [7], especially in severe and critical cases. The pathophysiological mechanism underlying thrombotic complications in Covid-19 patients is still largely unknown, but it is suspected that the excessive immune response generated upon coronavirus infection may trigger the thrombosis and coagulation pathway. In Covid-19, the incidence as well as the concentration of blood clots can be 2 or 3 times higher than the normal level [5].

Innate immune cells, i.e. neutrophils, macrophages, eosinophils and mast cells, our first line defence player against injury and pathogens, are not only capable of performing phagocytosis and releasing a huge variety of cytokines, but also able to extrude their web-like chromatin DNA fibers to extracellular space. These structures which were decorated with bioactive protein, are termed as extracellular traps (ETs) [8]. In the second decade since their discovery, ETs have become 
interesting features in the research field of noninfectious diseases such as atherosclerosis, thrombosis, autoimmunity, sepsis and even cancer [9].

This review presents current knowledge on the formation, properties and roles of ETs in human noninfectious diseases, particularly focusing on thrombotic inflammation. Subsequently, current findings on clinical and autopsy studies are discussed to briefly describe the immunopathology of Covid-19, including how SARSCoV2 may trigger the generation of ETs. These are then integrated to unveils the involvement of ETs in the immunopathological processes during Covid-19 infection. Lastly, this review summarizes the efforts on targeting the potential roles of ETs in Covid-19, either as a severity marker or as a therapeutic target.

\section{EXTRACELLULAR TRAPS AND IMMUNOTHROMBOSIS IN HUMAN}

\subsection{Formation of Extracellular Traps}

Nearly two decades ago, Brinkmann et al. [8] discovered that neutrophils, the major innate inflammatory cells, were able to release extracellularly a web-like chromatin DNA fibers, consisting of histones, nuclear proteins, and serine proteases. These so called as Neutrophil Extracellular Traps (NETs) function to trap and kill the bacteria [10]. Other innate immune cells such as macrophage, eosinophil and mast cell, are also then reported to generate similar traps, namely Macrophage Extracellular Traps (METs), Eosinophil Extracellular Traps (EETs) and Mast Cell Extracellular Traps (MCETs), respectively $[11,12]$. The generation of these traps also identifies a unique type of cell death namely (suicidal) etosis [13]. Nowadays, the origin cells could also survive after releasing the traps, a phenomenon later known as vital etosis [9].

Not only pathogens such as bacteria, fungi, parasites and viruses, but also different stimuli such as autoantibodies (Abs), activated platelets, cholesterol and monosodium urate crystals, also certain cytokines and chemokines could initiate ETs. Briefly, stimulated and activated innate inflammatory cells initiate the NADPH oxidase, producing reactive oxygen species (ROS) and activating peptidyl arginine deiminase 4 (PAD4), resulting in chromatin decondensation. The bioactive enzymes such as neutrophil elastase (NE), myeloperoxidase (MPO), or tryptase are migrated into the nucleus to promote further chromatin decondensation. When the nuclear membrane is disintegrated, the unfolded chromatin is released into the cytosol, where granular and cytosolic proteins are then attached. These combination of DNA, histones and granule proteins are extruded into the extracellular through the disrupted plasma membrane, marking what so called as 'suicidal' etosis because the dying cell dies. On the other hand, vital etosis presumably occurs via complement and without NADPH oxidase. Therefore,
PAD4 activation leading to chromatin decondensation takes place without the presence of ROS. The sequence of etosis events in vital etosis is similar with that in suicidal etosis, except for the expulsion of proteindecorated chromatin via vesicle and the cells of origin which are still alive after releasing the traps [14].

\subsection{Involvement of Extracellular Traps in Human Thromboinflammation Diseases}

Although ETs are beneficial to trap and kill pathogens, these structures could be detrimental in other clinical settings due to their pro-inflammatory, prothrombotic and pro-coagulant properties. When they are excessive and uncontrolled, ETs have the potential to propagate inflammation and thrombosis, leading to endothelial damage, impaired perfusion, tissue and organ damage as observed in patients with sepsis and ARDS [15].

\subsubsection{Pro-Inflammatory, Pro-Thrombotic and Pro-Coagulant Effects of Extracellular Traps}

The significant roles of ETs in acute and chronic inflammation have been acknowledged for instance NETs' contribution in regulating immune responses during autoimmune diseases, atherosclerotic inflammation and atherothrombosis [14]. Component of ETs such as chromatin DNA is a potent stimulus for dendritic cells (DCs) to synthesize type I interferon (IFN), which not only contains antimicrobial property but also stimulates autoimmunity in systemic lupus erythematosus (SLE) and psoriasis. Other NETs components such as cathepsin $\mathrm{G}$ and cathelicidins could attract and activate other immune cells such as monocytes and DCs in atherosclerosis. In turns, NETs stimulate macrophages to release cytokines, interleukin (IL) $1 \beta$ and IL6, recruiting more immune cells to the plaque [16]. Although etosis is a part of innate immune weapons, ETs could contribute to adaptive immunity via the activation of $\mathrm{T}$-cells, i.e. $\mathrm{T}$ helper 17 cells. The association of IL17 with NETs has been reported in psoriasis, atherosclerosis and atherothrombosis [17].

Several studies have reported the significance of ETs (mostly NETs) in thrombotic inflammation (also known as immunothrombosis). Fuchs et al. (2010) was first to report the pro-thrombotic properties of NETs, demonstrating that NETs provided a scaffold for activated platelets and erythrocyte adhesion, leading to thrombus aggregation, and thrombin formation. NETs are also pro-coagulant structures which are capable of stimulating the intrinsic and extrinsic coagulation pathways such as VWF (von Willebrand factor), fibronectin, fibrinogen, FXII (factor XII), and TF (tissue factor). All in all, NETs add strength and stability to the fibrin formation in thrombus so that the clots become thicker, stable and rigid, resulting to a significant prolonged clot lysis time. On the other hand, these prothrombotic and pro-coagulant effects of NETs exhibit a 
harmful risk of hypercoagulability, leading to vessel occlusion even in small vessels [18].

\subsubsection{Roles of Etosis in Thromboinflammatory Diseases: Evidence from Human Studies}

Etosis (mostly in the form of NETs) are involved in both arterial and venous thrombosis. The evidence of ETs in human arterial diseases arises from studies in patients with myocardial infarction (MI) and stroke. In ST-elevation myocardial infarction (STEMI), NETs were reported as a predictor for infarct size and resolution [19]. NETs were present in the atherosclerotic plaques with thrombotic complications but not in intact plaques, suggesting their roles in destabilizing plaque and aggravating atherothrombosis [20]. Also, NETs were mostly identified in both fresh and lytic, but not in organized coronary thrombi, suggesting their roles in thrombus growth and expansion [17]. Furthermore, NETs were also reported in the blood from culprit lesion site of MI [21], coronary stent thrombosis [22], and cerebral thrombi [23], the latter was associated with atrial fibrillation and all cause of mortality in ischemic stroke patients [15]. In deep venous thrombosis (DVT) and pulmonary embolism (PE), NETs were formed predominantly during the organizing stage of venous thrombosis development [24], indicating their putative roles in thrombus stabilization. Furthermore, circulating NET components were reported to be associated with disease severity in both arterial and venous thrombosis [25,26]. However, whether NETs are the cause or the consequence of thromboinflammation is still undetermined [27].

\section{IMMUNOPATHOGENESIS OF COVID- 19 AND THE EVIDENCE OF ETOSIS}

The global outbreak of Covid-19 caused by single stranded enveloped non-segmented RNA virus, namely severe acute respiratory syndrome-coronavirus (SARSCoV2), the pathology of which is not yet fully understood. Nevertheless, learning from the previous outbreak of SARS and MERS, immunopathogenesis of Covid-19 could be in a similar language [10]. In those two previous diseases, interaction between viral and host factors is the main driver of immune response against SARS - CoV and MERS-CoV infection [10,11].

It is already known that virus are able induce ETs, and in return, ETs are potent effectors of immune defence against the virus. However, the virus-induced etosis could still possess dangerous risk. In one hand, etosis are beneficial in destroying the virus, while on the other hand, etosis could trigger hyperactive inflammatory and immunological reactions as well as hypercoagulability. The evidence that etosis is present in Covid-19 and may contribute to the severe phases of Covid-19 have just recently emerged and thus attract the attention from the researchers and clinicians around the world [28,29].

\subsection{SARS-Cov2 Induces the Formation of (Neutrophil) Extracellular Traps}

Previous studies have documented the role of neutrophils and NETs in viral infections although the exact mechanisms are still not fully understood [14]. During human immunodeficiency virus (HIV)-1 infection, the release of ROS induces NETs formation, which in turn NETs could capture and neutralize HIV virions with their secretion of MPO and $\alpha$-defensins [30]. Similarly, NETs were also stimulated in response to human influenza $\mathrm{A}$, dengue and respiratory syncytial virus $[10,31]$. Therefore, it is assumed that SARS-CoV2 should also be able to stimulate NETs generation. Indeed, recent reports demonstrate that SARS-CoV2 is able to induce classical NETosis in vitro [32] and that in vitro healthy neutrophils could directly release NETs in a PAD-4-dependent manner when they are stimulated with viable SARS-CoV2 [33].

\subsection{The Immunopathological Mechanisms of Covid-19: Inflammation, Thrombosis and Coagulopathy}

As the first line defence mechanism, innate immunity plays a crucial role in fighting against SARSCOV2 infection and may link to the adaptive immune response, as such these open a strategic window for immune intervention. SARS-CoV2 infected patients have increased neutrophils and reduced total lymphocytes. A lung autopsy from a Covid-19 confirmed patient revealed massive neutrophil infiltration in pulmonary capillaries and alveolar space, showing acute inflammation of the entire respiratory tract [6]. SARS-CoV2 infection also directly attract macrophages and $\mathrm{T}$ cells, which are associated with the severe or lethal cases of Covid-19 [34]. Alongside the active viral replication, the increased influx of neutrophils and macrophages also results in an increased amount of pro-inflammatory cytokines such as IL1 $\beta$, IL2, IL6, IL7, IL8, IL10, IL17 and type I IFN $\gamma$.

On the other hand, the generated immune response could be excessive, termed as cytokine storm, which could lead to sepsis, ARDS, multiple organ damage and even death [12]. Hyperproduction of IFN $\gamma$ and increased C-Reactive Protein (CRP) were observed in severe Covid-19 patients. Furthermore, Covid-19 patients in ICU care had higher plasma levels of other innate cytokines such as tumour necrosis factor- $\alpha$ (TNF $\alpha)$, IL18 and chemokine MCP3 [35]. Cytokine storm is postulated to be responsible in the severe and death cases of Covid-19 younger patients without pre-existing conditions. Once the patient is cured, the associated damage i.e. pulmonary tissue could leave particular sequelae such as functional impairment and reduced lung capacity [12]. 
Furthermore, the hyperactivation of monocytes and neutrophils with their corresponding inflammatory cytokines leads to vascular endothelial injury and creates a prothrombotic state. It is believed that severe inflammatory process in the lungs of Covid-19 patients initiates a cascade of inflammatory thrombosis in the pulmonary vasculature, causing local and general coagulopathy and resulting to further macro- and microvascular thrombosis [1,36]. The thrombotic and coagulopathy events could appear as both venous (DVT and the most common PE) and arterial (MI and ischemic stroke) thrombosis, also other systemic thromboembolisms including disseminated intravascular coagulation (DIC), sepsis and multiple organ damage. These will reflect to increased levels of D-dimer, fibrinogen, factor VIII (FVIII) and von Willebrand factor (vWF), also decreased levels of antithrombin and TEG in Covid-19 patients at later stages.

\subsection{Clinical and Autopsy Findings Related to Etosis in Covid-19}

The first report on the association of etosis in Covid19 was obtained from the autopsy of lungs from open necropsies of Covid-19 patients [33]. They observed intense neutrophilic infiltration in pulmonary interstitial spaces and alveoli. Correspondingly, they proposed that NETs could play a prominent role in Covid-19. Indeed, plasma MPO-DNA complexes significantly increased in Covid-19 patients needed intubation and were associated with death as outcome [37]. Also, plasma platelet factor 4 (PF4) and RANTES, the trigger of netosis have increased. The same study also confirmed that pulmonary autopsies showed NET-containing microthrombi with neutrophil- platelet infiltration. NETs was also observed in another study on fixed lung tissue obtained from cohort Covid-19 patients, although the number of subjects was small [33]. In addition, NETs were found in tracheal aspirates of 12 patients on mechanical ventilation and in the plasma of those patients at a higher concentration. Similarly, Zuo et al. found higher numbers of serum cell-free DNA, DNAMPO complexes and citrullinated histone (CitH3) in 50 Covid-19 patients than in healthy controls [29]. Also, aggregated NETs were prominently observed inside the micro-vessels of kidney, liver and lungs, causing failure of these organs associated with endothelial damage [38]. All in all, these findings indicate the prominent presence of NETs in Covid-19 and that NETs are likely to contribute to the pathogenesis of Covid-19.

\section{POTENTIAL CONTRIBUTION OF EXTRACELLULAR TRAPS IN COVID-19}

It has been shown that SARS-CoV2 could activate NETosis in human neutrophils in vitro. The evidence for the presence of NETs (and etosis) in Covid-19 are limited, however it is likely that more findings on NETs could be reported in the near future since the pandemic still goes on. NETs contributing immunothrombosis are responsible for clinical presentations in patients with severe Covid-19 and may represent a potential target for severity biomarker and/ or therapeutic intervention [39].

\subsection{Potency of Etosis as A Severity Marker}

Previously, it has been reported that circulating NETs (and NETs components) are associated with severity of MI [25], sepsis [40] and acute lung injury [41]. Cell-free DNA is also strongly correlated with acute inflammatory markers including C-reactive protein and tissue damage markers such as D-dimer and lactate dehydrogenase [40]. Given the proinflammatory, pro-thrombotic and pro-coagulant properties of ETs [42], this structure could potentially be a severity marker during Covid-19 infection. Elevated NETs components such as cell-free DNA, MPO-DNA complexes and CitH3 [29,32] as well as Ddimer (tissue damage markers), prothrombin time and fibrin degradation products (hypercoagulability markers) have been observed in serum samples of severe Covid-19 patients, i.e. with ARDS [43]. Severe Covid-19 was also characterized by the prominent presence of NETs inside the pulmonary micro-vessels [32] and consistently increased NETs turnover marker [38]. Furthermore, it is known that disease severity also correlates with pro-inflammatory cytokines. In this regard, NETs-related cytokines were found in the lungs of Covid-19 patients, i.e. IL1 and TNFa [28,37]. Therefore, it is likely that ETs (and/ or their components) could be further considered as severity markers in the progression of Covid-19 patients.

\subsection{Casting Etosis as A Therapeutic Target}

As mentioned above, the severity of Covid-19 infection is presumably associated with the presence of NETs, thus, blocking NETs formation or destructing NETs structures hypothetically could be used to ameliorate NETs-mediated inflammatory and thrombotic tissue damage associated with Covid-19. Other than vaccine development, finding a potential method to block NETs formation and/ or to destroy the already formed NETs is currently being a hot topic in Covid-19-related research.

One of those strategies to prevent NETs formation is the inhibition of PAD4 and NE pathway. Previous study on atherosclerotic mice had shown that PAD4 can be inhibited by chloramidine treatment, thereby decreased atherosclerotic lesion size and delayed carotid artery thrombosis [44]. However, chloramidine lacks specificity which is harmful to be used in clinical setting. In Japan and South Korea, NE inhibitors, sivelestat was approved to treat ARDS but it did not increase survival after ARDS in a meta-analysis of 
clinical trials. A new generation of this drug type is currently under phase-1 trial [28].

Other than limiting NETs formation, destroying NETs structure could also be an option to prevent the dangerous effects of NETs [42]. DNase-1 could be used to dissolve NETs by breaking up the DNA strands. Application of DNase had previously shown to be useful in clearing neutrophil-rich bronchial mucous of cystic fibrosis patients and in improving MI following ischemia reperfusion injury [45,46]. Thus, DNase treatment may also prevent the further progression of Covid-19 to ARDS and restore normal perfusion of the heart and kidney microvasculature. It is expected that DNases may help dissolve the mucous secretions of Covid-19 patients, improving the lung function and the immunity in general [28].

Other strategy to affect NETs formation which had been previously studied, is the use of anti- coagulants and antiplatelet therapies. These two drugs have been studied in acute coronary syndrome but may not be sufficient to affect NETs formation [47]. In contrast, the use of heparin in Covid-19 showed that it effectively dissolves chromatin DNA in NETs and further inhibits their function as precipitators of inflammation [48]. Nevertheless, the effect of heparin should be tightly regulated with the appropriate dose, since NET-derived histones could interfere with the anti-coagulation effect of heparin.

Furthermore, researchers also pay attention to the development of drugs that prevent neutrophil recruitment which may effectively compromise the pathologic complications of lung and vascular injury in patients with Covid-19. Among these, vitamin $\mathrm{C}$ is considered due to its effects on neutrophil influx, activation, and NET-associated alveolar-capillary damage. Also, neonatal NET Inhibitory Factor (nNIF), a small protein found in umbilical cord blood of newborn babies, is also of consideration. In white blood cells treated with Covid-19 patient plasma, nNIF is reported to be able to diminish the hyperactive ETs activity [28].

\section{CONCLUSION}

Extracellular traps are potent weapon of innate immune cells, particularly neutrophil. The generation of NETs could play potential roles in the immunopathology of Covid-19, specifically during the severe and critical stages. Components of NETs have been found to be linked with the severity of Covid-19 and as such the development of novel therapeutic interventions targeting NETs could prevent the fatality of Covid-19. Targeting NETs (and etosis) in the therapeutic intervention of Covid-19 infection, however, should also be treated cautiously. Due to the lack of specificity, the treatment may danger the whole body since neutrophils (and the other inflammatory infiltrates) are still needed as the effectors of immune response. Therefore, further studies are required to elucidate the roles of ETs, both as a predictive biomarker and as a novel therapeutic target in severe Covid-19 cases.

\section{AUTHORS' CONTRIBUTIONS}

KRP designed the outline, searched for relevant literatures, conducted descriptive analytics review, prepared, wrote and checked the manuscript.

\section{ACKNOWLEDGMENTS}

The author would like to thank Prof. A.C. van der Wal and O. J. de Boer from the Department of Pathology, Amsterdam UMC University of Amsterdam for giving valuable advices, also Paramita C Kuswandi for providing feedback and helpful suggestions.

\section{REFERENCES}

[1] Abou-Ismail MY, Diamond A, Kapoor S, Arafah Y, Nayak L. The hypercoagulable state in COVID-19: Incidence, pathophysiology, and management. Thromb Res 2020;194:101-15. https://doi.org/10.1016/j.thromres.2020.06.029.

[2] Klok FA, Kruip MJHA, van der Meer NJM, Arbous MS, Gommers D, Kant KM, et al. Confirmation of the high cumulative incidence of thrombotic complications in critically ill ICU patients with COVID-19: An updated analysis. Thromb Res 2020:1-3.

https://doi.org/10.1016/j.thromres.2020.04.041.

[3] Middeldorp S, Coppens M, van Haaps TF, Foppen M, Vlaar AP, Müller MCA, et al. Incidence of venous thromboembolism in hospitalized patients with COVID-19. J Thromb Haemost 2020:19952002. https://doi.org/10.1111/jth.14888.

[4] Connors JM, Levy JH. COVID-19 and its implications for thrombosis and anticoagulation. Blood 2020;135:2033-40. https://doi.org/10.1182/blood.2020006000.

[5] Price LC, McCabe C, Garfield B, Wort SJ. Thrombosis and COVID-19 pneumonia: the clot thickens! Eur Respir J 2020:2001608. https://doi.org/10.1183/13993003.01608-2020.

[6] Ackermann M, Verleden SE, Kuehnel M, Haverich A, Welte T, Laenger F, et al. Pulmonary vascular endothelialitis, thrombosis, and angiogenesis in Covid-19. N Engl J Med 2020;383:120-8. https://doi.org/10.1056/NEJMoa2015432.

[7] Wise J. Covid-19 and thrombosis: what do we know about the risks and treatment? BMJ 2020;369:m2058.

https://doi.org/10.1136/bmj.m2058. 
[8] Brinkmann V, Reichard U, Goosmann C, Fauler B, Uhlemann Y, Weiss DS, et al. Neutrophil extracellular traps kill bacteria. Science (80- ) 2004;303:1532-5.

https://doi.org/10.1126/science.1092385.

[9] Brinkmann V. Neutrophil Extracellular Traps in the Second Decade. J Innate Immun 2018;10:414-21. https://doi.org/10.1159/000489829.

[10] Kaplan JM. Neutrophil extracelullar traps (NETs):Double-edged swords of innate immunity 1. J Immunol 2013;189:2689-95. https://doi.org/10.4049/jimmunol.1201719.Neutroph il.

[11] Goldmann O, Medina E. The expanding world of extracellular traps: Not only neutrophils but much more. Front Immunol 2012;3:1-10. https://doi.org/10.3389/fimmu.2012.00420.

[12] Pertiwi KR, de Boer OJ, Mackaaij C, Pabittei DR, de Winter RJ, Li X, et al. Extracellular traps derived from macrophages, mast cells, eosinophils and neutrophils are generated in a time-dependent manner during atherothrombosis. J Pathol 2019;247:505-12. https://doi.org/10.1002/path.5212.

[13] Wartha F, Henriques-Normark B. ETosis: a novel cell death pathway. Sci Signal 2008;1:pe25. https://doi.org/stke.121pe25 [pii] $\backslash n 10.1126 /$ stke.121 pe25.

[14] Jorch SK, Kubes P. An emerging role for neutrophil extracellular traps in noninfectious disease. Nat Med 2017;23:279-87. https://doi.org/10.1038/nm.4294.

[15] Thålin C, Hisada Y, Lundström S, Mackman N, Wallén H. Neutrophil Extracellular Traps: Villains and Targets in Arterial, Venous, and CancerAssociated Thrombosis. Arterioscler Thromb Vasc Biol 2019;39:1724-38. https://doi.org/10.1161/ATVBAHA.119.312463.

[16] Kaplan MJ, Radic M. Neutrophil Extracellular Traps: Double-Edged Swords of Innate Immunity. J Immunol 2012;189:2689-95. https://doi.org/10.4049/jimmunol.1201719.

[17] de Boer OJ, Li X, Teeling P, Mackaay C, Ploegmakers HJ, van der Loos CM, et al. Neutrophils, neutrophil extracellular traps and interleukin-17 associate with the organisation of thrombi in acute myocardial infarction. Thromb Haemost 2013;109:290-7. https://doi.org/10.1160/TH12-06-0425.

[18] Fuchs TA, Brill A, Duerschmied D, Schatzberg D, Monestier M. Extracellular DNA traps promote thrombosis 2010;107:15880-5. https://doi.org/10.1073/pnas.1005743107/-

/DCSupplemental.www.pnas.org/cgi/doi/10.1073/pn as. 1005743107 .
[19] Mangold A, Alias S, Scherz T, Hofbauer T, Jakowitsch J, Panzenböck A, et al. Coronary neutrophil extracellular trap burden and deoxyribonuclease activity in ST-elevation acute coronary syndrome are predictors of ST-segment resolution and infarct size. Circ Res 2015;116:118292.

https://doi.org/10.1161/CIRCRESAHA.116.304944.

[20] Pertiwi KR, Van Der Wal AC, Pabittei DR, Mackaaij C, Van Leeuwen MB, Li X, et al. Neutrophil Extracellular Traps Participate in All Different Types of Thrombotic and Haemorrhagic Complications of Coronary Atherosclerosis. Thromb Haemost 2018. https://doi.org/10.1055/s-00381641749.

[21] Mangold A, Alias S, Scherz T, Hofbauer T, Jakowitsch J, Panzenböck A, et al. Coronary neutrophil extracellular trap burden and deoxyribonuclease activity in ST-elevation acute coronary syndrome are predictors of ST-segment resolution and infarct size. Circ Res 2015;116:118292.

https://doi.org/10.1161/CIRCRESAHA.116.304944.

[22] Riegger J, Byrne RA, Joner M, Chandraratne S, Gershlick AH, Ten Berg JM, et al. Histopathological evaluation of thrombus in patients presenting with stent thrombosis. A multicenter European study: A report of the prevention of late stent thrombosis by an interdisciplinary global European effort consortium $\dagger$. Eur Heart J 2016;37:1538-49. https://doi.org/10.1093/eurheartj/ehv419.

[23] Laridan E, Denorme F, Desender L, François O, Andersson T, Deckmyn $\mathrm{H}$, et al. Neutrophil extracellular traps in ischemic stroke thrombi. Ann Neurol 2017;82:223-32. https://doi.org/10.1002/ana.24993.

[24] Savchenko AS, Martinod K, Seidman MA, Wong SL, Borissoff JI, Piazza G, et al. Neutrophil extracellular traps form predominantly during the organizing stage of human venous thromboembolism development. J Thromb Haemost 2014;12:860-70. https://doi.org/10.1111/jth.12571.

[25] Borissoff JI, Joosen IA, Versteylen MO, Brill A, Fuchs TA, Savchenko AS, et al. Elevated levels of circulating DNA and chromatin are independently associated with severe coronary atherosclerosis and a prothrombotic state. Arterioscler Thromb Vasc Biol 2013;33:2032-40. https://doi.org/10.1161/ATVBAHA.113.301627.

[26] Laridan E, Martinod K, De Meyer SF. Neutrophil Extracellular Traps in Arterial and Venous Thrombosis. Semin Thromb Hemost 2019. https://doi.org/10.1055/s-0038-1677040.

[27] Jiménez-Alcázar M, Kim N, Fuchs TA. Circulating Extracellular DNA: Cause or Consequence of Thrombosis? Semin Thromb Hemost 2017;43:553- 
61. https://doi.org/10.1055/s-0036-1597284.

[28] Barnes BJ, Adrover JM, Baxter-Stoltzfus A, Borczuk A, Cools-Lartigue J, Crawford JM, et al. Targeting potential drivers of COVID-19: Neutrophil extracellular traps. J Exp Med 2020;217:1-7. https://doi.org/10.1084/jem.20200652.

[29] Zuo Y, Yalavarthi S, Shi H, Gockman K, Zuo M, Madison JA, et al. Neutrophil extracellular traps in COVID-19. JCI Insight 2020;5. https://doi.org/10.1172/jci.insight.138999.

[30] Jenne CN, Kubes P. NETs tangle with HIV. Cell Host Microbe 2012;12:5-7. https://doi.org/10.1016/j.chom.2012.07.002.

[31] Matoszka N, Działo J, Tokarz-Deptuła B, Deptuła W. NET and NETosis - new phenomenon in immunology. Postepy Hig Med Dosw 2012;66:43745. https://doi.org/10.5604/17322693.1001178.

[32] Arcanjo A, Logullo J, Menezes CCB, Giangiarulo TC de SC, Barroso SPC, Todeschini A, et al. The Emerging Role of Neutrophil Extracellular Traps in Severe Acute Respiratory Syndrome Coronavirus 2 (COVID-19)\&nbsp; https://doi.org/10.21203/rs.3.rs-40461/v1.

[33] Veras FP, Pontelli M, Silva C, Toller-Kawahisa J, de Lima M, Nascimento D, et al. SARS-CoV-2 triggered neutrophil extracellular traps (NETs) mediate COVID-19 pathology. MedRxiv 2020:2020.06.08.20125823. https://doi.org/10.1101/2020.06.08.20125823.

[34] Prompetchara E, Ketloy C, Palaga T. Immune responses in COVID-19 and potential vaccines: Lessons learned from SARS and MERS epidemic. Asian Pacific J Allergy Immunol 2020;38:1-9. https://doi.org/10.12932/AP-200220-0772.

[35] Li G, Fan Y, Lai Y, Han T, Li Z, Zhou P, et al. Coronavirus infections and immune responses. J Med Virol 2020;92:424-32. https://doi.org/10.1002/jmv.25685.

[36] Colling ME, Kanthi Y. COVID-19-associated coagulopathy: An exploration of mechanisms. Vasc Med (United Kingdom) 2020. https://doi.org/10.1177/1358863X20932640.

[37] Middleton EA, He X-Y, Denorme F, Campbell RA, Ng D, Salvatore SP, et al. Neutrophil Extracellular Traps (NETs) Contribute to Immunothrombosis in COVID-19 Acute Respiratory Distress Syndrome. Blood 2020. https://doi.org/10.1182/blood.2020007008.

[38] Leppkes M, Knopf J, Naschberger E, Lindemann A, Singh J, Herrmann I, et al. Vascular occlusion by neutrophil extracellular traps in COVID-19. EBioMedicine 2020;58:102925. https://doi.org/10.1016/j.ebiom.2020.102925.
[39] Nathan C. Neutrophils and COVID-19: Nots, NETs, and knots. J Exp Med 2020;217:3-5. https://doi.org/10.1084/jem.20201439.

[40] Silk E, Zhao H, Weng H, Ma D. The role of extracellular histone in organ injury. Cell Death Dis 2017;8:e2812. https://doi.org/10.1038/cddis.2017.52.

[41] Caudrillier A, Kessenbrock K, Gilliss BM, Nguyen JX, Marques MB, Monestier M, et al. Platelets induce neutrophil extracellular traps in transfusionrelated acute lung injury. J Clin Invest 2012;122:2661-71.

https://doi.org/10.1172/JCI61303.ALI.

[42] Döring Y, Soehnlein O, Weber C. Neutrophil extracellular traps in atherosclerosis and atherothrombosis. Circ Res 2017;120:736-43. https://doi.org/10.1161/CIRCRESAHA.116.309692.

[43] Engelmann B, Massberg S. Thrombosis as an intravascular effector of innate immunity. Nat Rev Immunol $2013 ; 13: 34-45$ https://doi.org/10.1038/nri3345

[44] Knight JS, Luo W, O’Dell AA, Yalavarthi S, Zhao $\mathrm{W}$, Subramanian V, et al. Peptidylarginine deiminase inhibition reduces vascular damage and modulates innate immune responses in murine models of atherosclerosis. Circ Res 2014;114:94756.

https://doi.org/10.1161/CIRCRESAHA.114.303312.

[45] Martínez-Alemán SR, Campos-García L, PalmaNicolas JP, Hernández-Bello R, González GM, Sánchez-González A. Understanding the Entanglement: Neutrophil Extracellular Traps (NETs) in Cystic Fibrosis. Front Cell Infect Microbiol 2017;7:1-7. https://doi.org/10.3389/fcimb.2017.00104.

[46] Fadini GP, Menegazzo L, Scattolini V, Gintoli M, Albiero M, Avogaro A. A perspective on NETosis in diabetes and cardiometabolic disorders. Nutr Metab Cardiovasc Dis 2016;26:1-8. https://doi.org/10.1016/j.numecd.2015.11.008.

[47] Ylä-Herttuala S, Bentzon JF, Daemen M, Falk E, Garcia-Garcia HM, Herrmann J, et al. Stabilization of atherosclerotic plaques: An update. Eur Heart J 2013;34:3251-8. https://doi.org/10.1093/eurheartj/eht301.

[48] Tang N, Bai H, Chen X, Gong J, Li D, Sun Z. Anticoagulant treatment is associated with decreased mortality in severe coronavirus disease 2019 patients with coagulopathy. J Thromb Haemost 2020:10949. https://doi.org/10.1111/jth.14817. 